\title{
Endobronchial Ultrasound-Guided Transbronchial Biopsy of Peripheral Pulmonary Lesions: How Many Specimens Are Necessary?
}

\author{
Chun-Ta Huang a, b Yi-Ju Tsai ${ }^{\mathrm{c}}$ Wei-Yu Liao ${ }^{\mathrm{a}}$ Pei-Chen $\mathrm{Wu}^{\mathrm{d}}$ Chao-Chi Ho ${ }^{\mathrm{a}}$ \\ Chong-Jen $\mathrm{Yu}^{\mathrm{a}}$ Pan-Chyr Yang ${ }^{\mathrm{a}}$ \\ Departments of ${ }^{\mathrm{a}}$ Internal Medicine and ${ }^{\mathrm{b}}$ Traumatology, National Taiwan University Hospital, ${ }^{\mathrm{c} S c h o o l}$ of Medicine, \\ College of Medicine, Fu-Jen Catholic University, and ${ }^{\mathrm{d}}$ Center for Genomic Medicine, National Taiwan University \\ Hospital, Taipei, Taiwan, ROC
}

\section{Key Words}

Bronchoscopy $\cdot$ Endobronchial ultrasound $\cdot$ Peripheral pulmonary lesion $\cdot$ Specimen $\cdot$ Transbronchial biopsy

\begin{abstract}
Background: Although endobronchial ultrasound (EBUS)guided transbronchial biopsy (TBB) has been shown to increase the diagnostic yield over conventional bronchoscopic techniques, an important issue regarding the optimal number of biopsy specimens required has not been thoroughly investigated. Objectives: We sought to examine whether the number of biopsy specimens taken was associated with the diagnostic yield of EBUS-guided TBB and, if this was the case, to determine the optimal number of specimens required for the maximum diagnostic yield in peripheral pulmonary lesions. Methods: The medical records of patients undergoing EBUS-guided TBB for the diagnosis of peripheral pulmonary lesions from 2008 to 2010 were retrospectively reviewed. The association of clinical and radiological features, including the number of biopsy specimens, with the diagnostic yield was analysed. Results: A total of 384 patients were included for analysis. The overall diagnostic yield of EBUS-guided TBB was 73\%, and the only factor
\end{abstract}

influencing the diagnostic yield was the position of the probe. Patients in which the EBUS probe was placed within the lesions had a significantly higher yield (85\%) than those in which the probe was adjacent to or outside the lesions $(38 \% ; p<0.001)$. When the number of biopsy specimens was determined based on their adequacy, it was an insignificant factor in predicting the diagnostic yield. Conclusions: Probe position independently predicts the diagnostic yield of EBUS-guided TBB. In real-world practice, the optimal number of biopsy specimens should be decided on a case-bycase basis.

Copyright $\odot 2012$ S. Karger AG, Basel

\section{Introduction}

Radial probe endobronchial ultrasound (EBUS) employs a flexible catheter housing an ultrasound transducer which produces a 360-degree ultrasound image, and was first applied to guide transbronchial biopsy (TBB) by Herth et al. [1] in 2002. EBUS-guided TBB has an advantage over conventional bronchoscopic TBB and percutaneous computed tomography (CT)-guided biopsy of the lung in diagnosing peripheral pulmonary lesions (PPLs)

\section{KARGER}

Fax +41613061234

E-Mail karger@karger.ch

www.karger.com (c) 2012 S. Karger AG, Basel

$0025-7931 / 12 / 0842-0128 \$ 38.00 / 0$

Accessible online at:

www.karger.com/res
Chao-Chi Ho

Department of Internal Medicine

National Taiwan University Hospital

7 Chung-Shan South Road, Taipei 100, Taiwan (ROC)

Tel.+88622356 2905, E-Mail ccho1203@ntu.edu.tw 
because of a significantly higher diagnostic yield and an extremely favourable safety profile, respectively $[2,3]$. Over the past 10 years, several studies have been conducted to examine the influence of specific clinical and radiological features on the diagnostic performance of EBUS-guided TBB $[2,4]$. However, an important issue regarding the number of biopsy specimens required per patient has not been vigorously investigated. The only study dealing with this issue recommended that at least 5 biopsy specimens should be obtained by TBB using EBUS [5].

Accordingly, the purpose of this study was to examine whether the number of biopsy specimens taken was associated with the diagnostic yield of EBUS-guided TBB, and if this was the case, to determine the optimal number of specimens required for the maximum diagnostic yield in PPLs.

\section{Materials and Methods}

Study Subjects

The medical records of 2,988 patients referred to the National Taiwan University Hospital (Taipei, Taiwan) for bronchoscopy from January 1, 2008, to December 31, 2010, were retrospectively reviewed. A total of 384 patients with PPLs who underwent EBUSguided TBB were included for analysis. PPLs were defined as lesions being surrounded by pulmonary parenchyma without bronchoscopic evidence of endobronchial abnormalities. Since late 2007, no patients with PPLs have undergone conventional TBB at our institution. This study was approved by the National Taiwan University Hospital Research Ethics Committee.

\section{Data Collection}

All patient records were independently reviewed by two pulmonologists. Data extracted included patient demographics, final diagnosis, complications, duration of bronchoscopic procedures, number of biopsy specimens, auxiliary bronchoscopic diagnostic procedures and position of the EBUS probe. Post-biopsy haemorrhage was defined as bleeding needing further intervention or intensive care observation; self-limited bleeding was not regarded as a complication. Given the extremely favourable safety profile of EBUS-guided TBB, a chest x-ray was obtained only if clinically indicated. The duration of bronchoscopy was defined as the time period from initial bronchoscope insertion to final bronchoscope withdrawal. The position of the probe relative to the target PPLs was classified as either within, adjacent to or outside the lesion, as previously reported [6]. Patients whose diagnosis could not be achieved through bronchoscopy underwent percutaneous CTguided biopsy, surgery or clinical/radiological follow-up to obtain a final diagnosis. All CT sections of the chest were also reviewed by two of the authors and the largest diameters in the cross-sectional plane, location and characteristics of the PPLs were recorded. When the PPL overlapped two lobes of the lung it was assigned to the lobe that contained greater than one half of the area of the PPL. In order to assess any association between the number and size of the biopsy specimens, pathology reports were retrieved and the mean diameter of the largest specimen for each patient was recorded.

\section{Bronchoscopic Technique}

The whole procedure was performed by pulmonary fellows under the supervision of experienced pulmonologists. An Olympus BF-P260F video bronchoscope (Olympus, Tokyo, Japan) with a $2.0-\mathrm{mm}$ working channel and an endoscopic ultrasound system (EU-M30S; Olympus), equipped with a $20-\mathrm{MHz}$ radialtype flexible probe (UM-S20-20R; Olympus) was used. Bronchoscopic procedures were performed after local anaesthesia with $5 \mathrm{ml}$ of $2 \%$ nebulized lidocaine and intramuscular administration of meperidine $50 \mathrm{mg}$. In addition, one or two aliquots of $1 \mathrm{ml}$ of $2 \%$ lidocaine were instilled over the mucosa of the larynx, carina and bronchial tree during bronchoscopy. EBUSguided TBB was performed as previously described [7]. The biopsy was repeated until two adequate specimens, defined as lung specimens spilling over the surface of the biopsy forceps, were collected, and a total of at least three specimens were obtained. Bronchial washing, brushing or both were performed after TBB based on the discretion of the specialist pulmonologist in charge. While all specimens obtained by bronchial washing, brushing and TBB were submitted for pathologic examination, only bronchial washing specimens were submitted for bacteriological studies, including smears and cultures for bacteria, fungi and mycobacteria.

\section{Statistical Methods}

Comparisons were made using the Student $t$ test or one-way analysis of variance (ANOVA) for continuous variables, and the $\chi^{2}$ test or Fisher's exact test for categorical variables. All variables with a $\mathrm{p}$ value $<0.1$ in the univariate analysis were entered into multivariate logistic regression analysis. A two-tailed $\mathrm{p}$ value $<0.05$ was considered to be statistically significant. All statistical analyses were performed using statistical software (version 15; SPSS, Chicago, Ill., USA).

\section{Results}

Of the 384 patients analysed in this study, 220 (57\%) were male, the mean age was 64.7 years (range 21-100) and the mean \pm standard deviation diameter of the PPLs was $38 \pm 16 \mathrm{~mm}$. The mean duration of the bronchoscopic procedures was $23.9 \pm 7.1 \mathrm{~min}$. The PPLs were located in the right upper lobe in 104 patients (27\%), the right middle lobe in 48 patients (13\%), the right lower lobe in 71 patients (18\%), the left upper lobe in 98 patients (26\%) and the left lower lobe in 63 patients (16\%). The CT characteristics of the PPLs indicated 338 (88\%) solid lesions, 19 (5\%) cavity lesions, 12 (3\%) ground-glass opacity lesions and 15 (4\%) mixed ground-glass opacity lesions. The probe position was described as being within the PPLs in 280 patients (73\%), adjacent to the PPLs in 86 patients (22\%) and outside the PPLs in 18 patients (5\%). Five 
hundred and nineteen auxiliary bronchoscopic procedures were performed, including bronchial washing and brushing in 364 and 155 patients, respectively. The overall complication rate was $2 \%$ (9/384 patients). Two patients experienced expectoration of more than $100 \mathrm{ml}$ of blood within $1 \mathrm{~h}$ of the procedure and required intensive care observation. They were treated with endobronchial topical application of epinephrine through the bronchoscope and intravenous administration of tranexamic acid. Both of them had a good recovery. Seven patients had post-procedure pneumothorax, 3 of whom underwent simple aspiration and the remaining 4 patients required only observation and supplemental oxygen therapy. No mortality occurred during the bronchoscopic procedures.

Table 1 summarizes the final diagnoses of the 384 patients. A definitive diagnosis was achieved by means of EBUS-guided TBB in 279 (73\%) patients. Of the 105 patients with a non-diagnostic TBB, the diagnosis was primarily established by percutaneous CT-guided biopsy $(40 / 105,38 \%)$, surgery $(31 / 105,30 \%)$ and clinical/radiological follow-up (18/105, 17\%). Overall, 294 (77\%) patients had malignant diseases and the majority (261/294, $89 \%$ ) of those were primary lung cancer. The most common diagnosis in patients with benign diseases was tuberculosis $(25 / 90,28 \%)$, reflecting the high tuberculosis burden in Taiwan.

The features associated with the diagnostic yield of EBUS-guided TBB are shown in table 2. Factors that significantly influenced the diagnostic yield of EBUS-guided TBB were the diameter of the lesion $(\mathrm{p}=0.003)$, location of the lesion on CT sections $(p=0.017)$, position of the probe on the EBUS image $(\mathrm{p}<0.001)$ and final diagnosis $(p=0.002)$. There were no significant differences in the diagnostic yield for the CT characteristics of the PPLs, development of complications, number of biopsy specimens and performance of additional bronchoscopic procedures.

In the univariate analysis, the diagnostic yield of the PPLs with a diameter of $30 \mathrm{~mm}$ or less $(97 / 153,63 \%)$ was significantly lower than that of PPLs with a diameter of more than $30 \mathrm{~mm}(182 / 231,79 \%$; $\mathrm{p}=0.001)$. PPLs in which the EBUS probe was placed within the PPL had a higher diagnostic yield $(239 / 280,85 \%)$ compared to those in which the probe was placed adjacent to or outside the PPL $(40 / 104,38 \%$; $<<0.001)$. The diagnostic yield for malignant PPLs (225/294, 77\%) was significantly higher than that for benign PPLs $(54 / 90,60 \%$; $\mathrm{p}=0.002$ ). PPLs of the upper lobes of the lungs tended to predict a higher diagnostic yield (155/202, 77\%) than
Table 1. Diagnostic methods and final diagnoses of 384 patients

\begin{tabular}{lc}
\hline & $\mathrm{n}(\%)$ \\
\hline $\begin{array}{l}\text { Malignant disease }(n=294) \\
\text { Final diagnosis }\end{array}$ \\
Adenocarcinoma & $179(61)$ \\
Squamous cell carcinoma & $39(13)$ \\
Non-small cell carcinoma & $24(8)$ \\
Metastasis & $23(8)$ \\
Small cell carcinoma & $19(6)$ \\
Lymphoma & $8(3)$ \\
Neuroendocrine carcinoma & $1(1)$ \\
Sarcoma & $1(1)$ \\
Diagnostic method & \\
EBUS TBB & $225(77)$ \\
Percutaneous CT-guided biopsy & $38(13)$ \\
Surgery & $19(6)$ \\
Biopsy from other organs & $9(3)$ \\
Bronchial brushing & $3(1)$
\end{tabular}

Benign disease $(n=90)$

Final diagnosis

Tuberculosis $25(28)$

Inflammatory change $18(20)$

Organizing pneumonia $6(7)$

Cryptococcosis $5(6)$

Pneumonia $4(4)$

Fibrosis $4(4)$

Sclerosing haemangioma $3(3)$

Lung abscess 3 (3)

Nontuberculous mycobacteriosis $2(2)$

Sarcoidosis $2(2)$

Pulmonary infarct 1 (1)

Hamartoma $1(1)$

Haematoma $1(1)$

Mycetoma $1(1)$

Adenoma 1 (1)

Lesion decreased or vanished 13 (14)

Diagnostic method

EBUS TBB $54(60)$

Clinical/radiologic follow-up $18(20)$

Surgery 12 (13)

Bronchial washing 3 (3)

Percutaneous CT-guided biopsy $2(2)$

Serology

$1(1)$

those of non-upper lobe regions of the lungs (124/182, $68 \% ; \mathrm{p}=0.059$ ). A multivariate logistic regression model was used to investigate independent factors (table 3) and only probe position was identified as a statistically significant factor predicting the diagnostic yield of EBUS-guided TBB $(\mathrm{p}<0.001)$. Lesion size, lesion location and final diagnosis did not influence the diagnostic yield of EBUS-guided TBB. 
Table 2. Factors associated with diagnostic yield of EBUS-guided TBB

\begin{tabular}{|c|c|c|c|}
\hline Characteristic & $\begin{array}{l}\text { Lesion } \\
\text { diagnosed by } \\
\text { EBUS TBB } \\
(\mathrm{n}=279)\end{array}$ & $\begin{array}{l}\text { Lesion not } \\
\text { diagnosed by } \\
\text { EBUS TBB } \\
(\mathrm{n}=105)\end{array}$ & $\begin{array}{l}\mathrm{p} \\
\text { value }\end{array}$ \\
\hline Age, years & $65 \pm 13$ & $64 \pm 12$ & 0.686 \\
\hline Male gender & $159(57)$ & $61(58)$ & 0.845 \\
\hline \multicolumn{4}{|l|}{ Lesion size, $\mathrm{mm}$} \\
\hline$\leq 20$ & $26(9)$ & $18(17)$ & \multirow[t]{3}{*}{0.003} \\
\hline$>20$ and $\leq 30$ & $71(25)$ & $38(36)$ & \\
\hline$>30$ & $182(65)$ & $49(47)$ & \\
\hline \multicolumn{4}{|l|}{ Lesion location } \\
\hline Right upper lobe & $86(31)$ & $18(17)$ & \multirow[t]{5}{*}{0.017} \\
\hline Right middle lobe & $28(10)$ & $20(19)$ & \\
\hline Right lower lobe & $54(19)$ & $17(16)$ & \\
\hline Left upper lobe & $69(25)$ & $29(28)$ & \\
\hline Left lower lobe & $42(15)$ & $21(20)$ & \\
\hline \multicolumn{4}{|c|}{ Characteristic on CT sections } \\
\hline Solid & $248(89)$ & $90(86)$ & \multirow[t]{2}{*}{0.393} \\
\hline Others $^{1}$ & $31(11)$ & $15(14)$ & \\
\hline \multicolumn{3}{|l|}{ Position of the probe } & \multirow[t]{4}{*}{$<0.001$} \\
\hline Within & $239(86)$ & $41(39)$ & \\
\hline Adjacent to & $35(13)$ & $51(49)$ & \\
\hline Outside & $5(2)$ & $13(12)$ & \\
\hline \multicolumn{4}{|l|}{ Final diagnosis } \\
\hline Malignant & $225(81)$ & $69(66)$ & \multirow[t]{2}{*}{0.002} \\
\hline Benign & $54(19)$ & $36(34)$ & \\
\hline \multicolumn{4}{|l|}{ Complication } \\
\hline Haemorrhage & $1(1)$ & $1(1)$ & 0.473 \\
\hline Pneumothorax ${ }^{2}$ & $6(3)$ & $1(1)$ & 0.677 \\
\hline \multicolumn{4}{|l|}{ No. of biopsy specimens } \\
\hline 3 & $101(36)$ & $48(46)$ & \multirow[t]{5}{*}{0.303} \\
\hline 4 & $64(23)$ & $15(14)$ & \\
\hline 5 & $43(15)$ & $15(14)$ & \\
\hline 6 & $38(14)$ & $13(12)$ & \\
\hline$\geq 7$ & $33(12)$ & $14(13)$ & \\
\hline \multicolumn{4}{|c|}{ Auxiliary diagnostic procedure } \\
\hline Bronchial washing 3 & $267(98)$ & $97(95)$ & 0.179 \\
\hline Bronchial brushing ${ }^{4}$ & $117(42)$ & $38(36)$ & 0.306 \\
\hline
\end{tabular}

Data are presented as mean \pm standard deviation or $\mathrm{n}$ with percentages in parentheses.

${ }^{1}$ Cavity, mixed ground-glass opacity and pure ground-glass opacity. ${ }^{2} 271$ patients having a chest X-ray within 3 days after bronchoscopy were included in the analysis. ${ }^{3} 364$ patients undergoing bronchial washing were included in the analysis. ${ }^{4} 155$ patients undergoing bronchial brushing were included in the analysis.

A total of 1,733 biopsy specimens were obtained via EBUS-guided TBB, and the mean number of specimens taken was $4.5 \pm 1.7$. The number of biopsy specimens retrieved per patient were as follows: 3 in 149 (39\%) patients, 4 in $79(21 \%)$ patients, 5 in $58(15 \%)$ patients, 6 in
$51(13 \%)$ patients and at least 7 in $47(12 \%)$ patients. The average diameters of the largest specimens for patients having 3, 4, 5, 6 and 7 or more biopsy specimens were 1.4 $\pm 0.4,1.6 \pm 0.5,1.6 \pm 0.6,1.6 \pm 0.4$ and $1.6 \pm 0.5 \mathrm{~mm}$, respectively $(\mathrm{p}=0.064)$. The diagnostic yield of EBUSguided TBB according to the number of biopsy specimens is presented in table 4. Overall, no differences in the diagnostic yield were observed based on the number of specimens. After categorizing the PPLs by lesion size, lesion location, position of the probe or final diagnosis, the number of biopsy specimens remained an insignificant factor in predicting the diagnostic yield.

\section{Discussion}

To the best of our knowledge, this is the largest study to investigate the association between the number of biopsy specimens and the diagnostic yield of EBUS-guided TBB. We found that there were no differences in the diagnostic yield according to the number of specimens obtained by TBB, and that probe position was the only characteristic significantly associated with the diagnostic yield of TBB using EBUS.

Obtaining more biopsy specimens adds some cost to the examination, because more pathology time is needed to process and read the specimens. In addition, unnecessary biopsies may prolong the examination time and pose additional risks for the development of post-biopsy complications. However, the maximum diagnostic yield of EBUS-guided TBB may not be achieved because of an insufficient number of specimens. Therefore, operators should compare the risks and benefits of TBB to obtain a higher number of biopsies with the results of other diagnostic procedures. Currently, there are no clear recommendations as to the optimal number of biopsy specimens that should be taken by TBB using EBUS. Yamada et al. [5] analysed 85 pathologically diagnosed PPLs and found that five biopsy specimens provided a diagnostic yield of $95 \%$ or higher, and the increment in the cumulative diagnostic yield was similar with regard to the diagnosis, size of the PPLs and position of the probe. The present study specifically investigated the influence of the number of biopsy specimens on the diagnostic yield of EBUS-guided TBB, however no relationship was found. In our daily bronchoscopic practice, the operators perform as many biopsies as they judge necessary to obtain adequate specimens to provide a definitive pathologic diagnosis for a given patient. Because of the differences in the experience and skill of the operators [8], this may be 
Table 3. Logistic regression analysis of factors on diagnostic yield of EBUS TBB

\begin{tabular}{lllr}
\hline Characteristic & & \multicolumn{2}{l}{ Multivariate } \\
\cline { 3 - 4 } & HR (95\% CI) & p value \\
\hline Lesion size & $>30$ mm versus $\leq 30 \mathrm{~mm}$ & $1.27(0.737-2.192)$ & 0.388 \\
Lesion location & upper lobe versus non-upper lobe & $1.44(0.858-2.410)$ & 0.168 \\
Probe position & within versus adjacent to or outside & $8.50(5.003-14.435)$ & $<0.001$ \\
Final diagnosis & malignant versus benign & $1.61(0.889-2.934)$ & 0.116 \\
\hline
\end{tabular}

$\mathrm{HR}=$ Hazard ratio; $\mathrm{CI}=$ confidence interval.

Table 4. Diagnostic yield of EBUS TBB according to the number of biopsy specimens

\begin{tabular}{|c|c|c|c|c|c|}
\hline \multirow[t]{2}{*}{ Characteristic } & \multicolumn{5}{|c|}{ Number of biopsy specimens } \\
\hline & 3 & 4 & 5 & 6 & $\geq 7$ \\
\hline All lesions & $101 / 149(68)$ & $64 / 79(81)$ & $43 / 58(74)$ & $38 / 51(75)$ & $33 / 47(70)$ \\
\hline \multicolumn{6}{|l|}{ Lesion size } \\
\hline$\leq 30 \mathrm{~mm}$ & $36 / 65(55)$ & $23 / 28(82)$ & $10 / 19(53)$ & $13 / 20(65)$ & $15 / 21(71)$ \\
\hline$>30 \mathrm{~mm}$ & $65 / 84(77)$ & $41 / 51(80)$ & $33 / 39(85)$ & $25 / 31(81)$ & $18 / 26(69)$ \\
\hline \multicolumn{6}{|l|}{ Lesion location } \\
\hline Upper lobe & $56 / 79(71)$ & $31 / 37(84)$ & $24 / 33(73)$ & $28 / 33(85)$ & $16 / 20(80)$ \\
\hline Non-upper lobe & $45 / 70(64)$ & $33 / 42(79)$ & $19 / 25(76)$ & $10 / 18(56)$ & $17 / 27(63)$ \\
\hline \multicolumn{6}{|l|}{ Position of the probe } \\
\hline Within & $87 / 104(84)$ & $52 / 56(93)$ & $39 / 47(83)$ & $33 / 36(92)$ & $28 / 37(76)$ \\
\hline Adjacent to or outside & $14 / 45(31)$ & $12 / 23(52)$ & $4 / 11(36)$ & $5 / 15(33)$ & $5 / 10(50)$ \\
\hline \multicolumn{6}{|l|}{ Final diagnosis } \\
\hline Malignant & $80 / 110(73)$ & $52 / 63(83)$ & $36 / 45(80)$ & $31 / 42(74)$ & $26 / 34(76)$ \\
\hline Benign & $21 / 39(54)$ & $12 / 16(75)$ & $7 / 13(54)$ & $7 / 9(78)$ & $7 / 13(54)$ \\
\hline
\end{tabular}

Data are presented as number of diagnosis achieved/total number of patients undergoing EBUS TBB (\%).

a more reasonable and realistic way to determine the number of biopsy specimens. Although an adequate specimen is usually determined according to the appearance, colour, consistency and size, its definition remains somewhat arbitrary and subjective in our institution. However, previous work has demonstrated that a diagnostic biopsy specimen will likely be obtained if the size of the specimen fills the forceps [9] and this is in accordance with the adequacy criteria adopted by our pulmonologists. Moreover, a consistent judgment of the biopsy specimens was observed in our study because the diagnostic yield of the PPLs and the average diameter of the largest biopsy specimens were similar irrespective of the number of specimens. On-site assessment of the biopsy specimens is performed at certain institutions and might allow the operators to immediately determine the quality of each biopsy specimen [10]. The use of on-site assessment would, however, increase the cost, prolong the procedure time and be labour consuming, and it is probably not feasible for the majority of hospitals worldwide. In line with a previous study [5], the diagnostic yield of TBB using EBUS differed among underlying diseases, probe position, lesion location and lesion size (table 4), but it was still unaffected by the number of biopsy specimens in these features. Thus, the results of this study suggest that the optimal number of biopsy specimens should be determined individually according to the adequacy of the retrieved specimens rather than a single predetermined number in real-world practice. However, given the retrospective nature of this study, it is difficult to further specify the criteria of an adequate specimen of EBUS-guided $\mathrm{TBB}$ and more work is warranted. 
The position of the probe is a universally recognized predictor of the diagnostic yield of TBB using EBUS [5-7, $11,12]$. After controlling for potential confounders, it was the only significant factor predicting the diagnostic yield in the present study. When the probe was placed within the PPL, a diagnostic yield of $85 \%$ was achieved. This again emphasizes the importance of introducing the probe within the PPL on the EBUS image before embarking on TBB. If the EBUS probe was only placed adjacent to or outside the PPL, the diagnostic yield of EBUS-guided TBB markedly decreased to less than $40 \%$ in this study. A similarly low diagnostic yield (23-42\%) for this kind of probe placement has been observed in other studies investigating the influence of probe position on the diagnostic yield of TBB using EBUS [5-7, 11, 12]. Under such circumstances, alternative or auxiliary approaches, such as percutaneous CT-guided biopsy or adding transbronchial needle aspiration to TBB $[12,13]$, should be considered to give the best diagnostic yield for PPLs.

The diagnosis $[11,14]$, lobar location $[5,6,14,15]$ and size $[6,15]$ of the PPLs have been shown to be factors influencing the diagnostic yield of EBUS-guided TBB in several studies; however, this was not the case in the present study. Although this discrepancy may be explained by differences in study design and the patient population studied, more probably it is because probe position is an important confounder not taken into account by these studies. In univariate analysis, we also found that the diagnostic yield of EBUS-guided TBB was determined by lesion size, lesion location and nature of the PPLs. In other words, as we took probe position into consideration, the above variables were no longer independent factors influencing the diagnostic yield. A similar finding has been reported in two other studies $[5,7]$. Thus, studies of this kind need to be interpreted cautiously and wisely.

There are some potential limitations to this study. First, inter-operator variability was difficult to analyse because the bronchoscopic procedures were always accomplished by a team. However, highly experienced pulmonologists supervised the entire process and this might minimize the inter-operator variability. Second, although the patients who underwent EBUS-guided TBB were always prospectively tracked by pulmonary fellows for the final diagnosis and development of complications, biases typically associated with retrospective studies, such as selection and ascertainment biases, could not be completely eliminated. Third, the present study was conducted in a tertiary care referral centre and the results may not be generalizable to other hospital settings.

In summary, based on our analysis of a large patient population, we suggest that the optimal number of biopsy specimens should be decided according to their adequacy in daily clinical practice, although further investigations are necessary to establish objectively defined criteria for adequate specimens. Furthermore, the position of the probe was the only factor predicting the diagnostic yield of EBUS-guided TBB and operators should try to obtain a favourable EBUS image prior to performing TBB.

\section{Financial Disclosure and Conflicts of Interest}

This work was supported by a grant from the Taiwan National Science Council (NSC-100-2321-B-002-058), which had no role in the study design, in the collection, analysis and interpretation of data, in the writing of the manuscript or in the decision to submit the manuscript for publication.

\section{References}

$\checkmark 1$ Herth FJ, Ernst A, Becker HD: Endobronchial ultrasound-guided transbronchial lung biopsy in solitary pulmonary nodules and peripheral lesions. Eur Respir J 2002;20:972974.

2 Steinfort DP, Khor YH, Manser RL, Irving LB: Radial probe endobronchial ultrasound for the diagnosis of peripheral lung cancer: systematic review and meta-analysis. Eur Respir J 2011;37:902-910.
- 3 Eberhardt R, Morgan RK, Ernst A, Beyer T, Herth FJ: Comparison of suction catheter versus forceps biopsy for sampling of solitary pulmonary nodules guided by electromagnetic navigational bronchoscopy. Respiration 2010;79:54-60.

4 Anantham D, Koh MS, Ernst A: Endobronchial ultrasound. Respir Med 2009;103: 1406-1414.
5 Yamada N, Yamazaki K, Kurimoto N, Asahina H, Kikuchi E, Shinagawa N, Oizumi S, Nishimura M: Factors related to diagnostic yield of transbronchial biopsy using endobronchial ultrasonography with a guide sheath in small peripheral pulmonary lesions. Chest 2007;132:603-608.

6 Kurimoto N, Miyazawa T, Okimasa S, Maeda A, Oiwa H, Miyazu Y, Murayama M: Endobronchial ultrasonography using a guide sheath increases the ability to diagnose peripheral pulmonary lesions endoscopically. Chest 2004;126:959-965. 
7 Huang CT, Ho CC, Tsai YJ, Yu CJ, Yang PC: Factors influencing visibility and diagnostic yield of transbronchial biopsy using endobronchial ultrasound in peripheral pulmonary lesions. Respirology 2009;14:859-864.

8 Stather DR, Maceachern P, Rimmer K, Hergott CA, Tremblay A: Validation of an endobronchial ultrasound simulator: differentiating operator skill level. Respiration 2011; $81: 325-332$

$\checkmark$ Curley FJ, Johal JS, Burke ME, Fraire AE: Transbronchial lung biopsy: can specimen quality be predicted at the time of biopsy? Chest 1998;113:1037-1041.

10 Diette GB, White P Jr, Terry P, Jenckes M, Rosenthal D, Rubin HR: Utility of on-site cytopathology assessment for bronchoscopic evaluation of lung masses and adenopathy. Chest 2000;117:1186-1190.
11 Chung YH, Lie CH, Chao TY, Wang YH, Lin AS, Wang JL, Lin MC: Endobronchial ultrasonography with distance for peripheral pulmonary lesions. Respir Med 2007;101:738745.

12 Chao TY, Chien MT, Lie CH, Chung YH, Wang JL, Lin MC: Endobronchial ultrasonography-guided transbronchial needle aspiration increases the diagnostic yield of peripheral pulmonary lesions: a randomized trial. Chest 2009;136:229-236.
3 Wahidi MM, Govert JA, Goudar RK, Gould MK, McCrory DC, American College of Chest Physicians: Evidence for the treatment of patients with pulmonary nodules: when is it lung cancer? ACCP evidence-based clinical practice guidelines (2nd edition). Chest 2007;132:94S-107S.

14 Eberhardt R, Anantham D, Ernst A, FellerKopman D, Herth F: Multimodality bronchoscopic diagnosis of peripheral lung lesions: a randomized controlled trial. Am J Respir Crit Care Med 2007;176:36-41.

15 Yoshikawa M, Sukoh N, Yamazaki K, Kanazawa K, Fukumoto S, Harada M, Kikuchi E, Munakata M, Nishimura M, Isobe $\mathrm{H}$ : Diagnostic value of endobronchial ultrasonography with a guide sheath for peripheral pulmonary lesions without $\mathrm{x}$-ray fluoroscopy. Chest 2007;131:1788-1793. 